\title{
Medium Access Control design for UWB Communication Systems: review and trends
}

\author{
Luca De Nardis, Maria-Gabriella Di Benedetto
}

\begin{abstract}
Future wireless networks are expected to achieve high bit rates at low cost, enabling multimedia and QoS-based services over the wireless medium. The Impulse Radio Ultra-wide Band (IR-UWB) technique is a promising candidate in the deployment of such networks, thanks to its potential robustness and capacity. In the past, most of the UWB research focused on hardware and physical layer aspects in order to solve the technological challenges posed by IR-UWB. UWB peculiar characteristics may, however, also stimulate innovative higher layers' design. This work addresses MAC issues for UWB Communication systems. Key areas such as medium sharing, MAC organization, packet scheduling and power control are reviewed. The impact of UWB on the above functions is discussed, and areas which require UWB specific design are identified. Finally, novel MAC functions enabled by UWB specific features, i.e. precise ranging and positioning, are presented.
\end{abstract}

Index Terms: UWB, MAC, Network Organization.

\section{INTRODUCTION}

The design of a Communication System traditionally proceeds along the principle of a layered architecture. The aim is to design each layer of the system independently of the internal structure of the lower layers, which are considered as black boxes offering a service to upper layers [1]. Rules of interaction between different layers are defined by interfaces which determine the requested inputs and corresponding outputs. Within this framework, the MAC is generally considered as the bottom part of the Data Link Control (DLC) layer. The service offered by the MAC to the upper DLC is to provide a bit pipe, preventing or resolving contentions in the access to the medium. Following the layered approach, the functions executed in the MAC should be defined without taking into account the underlying physical layer. The design of an efficient MAC often requires however an accurate knowledge of the physical layer, and in most existing systems specific properties of the transmission technique are exploited in order to reduce the effect of multiple access interference. In the case of Ultra-wide Band (UWB) systems, this is a crucial issue where UWB potentials (for example precise ranging capability) may enable the definition of novel MAC functions, as well as lead to a drastically different implementation of more traditional MAC functions.

As a general principle, the role of the MAC layer is to allow multiple users to share a common resource. The definition of resource, and of the procedures by which access to the medium is granted, depends on the adopted transmission and multiple

Manuscript received March 22, 2002; approved for publication by Raouf Boutaba, Division III Editor, June 11, 2003.

L. De Nardis and M.G. Di Benedetto are with the INFOCOM Department at the Università degli Studi di Roma La Sapienza, Rome, Italy.

E-mails: lucadn@newyork.ing.uniroma1.it and gaby@acts.ing.uniroma1.it. access techniques. As discussed above, the evaluation metrics for a MAC protocol should be however defined independently of the physical layer.

A tentative set of key parameters can be given as follows [2]:

- Throughput: percentage of channel capacity in use during data transmission

- Delay: average time spent by a packet in the MAC queue

- Fairness: equal opportunity for terminals to access the medium

Other parameters which are not listed above are usually related to a specific MAC protocol or a specific scenario. In a centralized architecture, for example, an evaluation criterion may consist in the degree of asymmetric flexibility in the bandwidth allocation to the downlink and uplink streams for a given user [3].

Accordingly, key MAC design objectives should be: i) to maximize throughput, ii) to guarantee an acceptable delay, and iii) to grant fair access. The above goals should be fulfilled in a dynamic environment, i.e. under variable channel conditions, traffic characteristics, and local network topologies. Flexibility is thus an additional feature which an advanced MAC should incorporate.

In this paper, we will analyze how the above objectives can be pursued in the case of UWB wireless networks. It should be noted that several issues being raised in the design of a MAC for UWB networks are not novel, and that similar problems have been solved in the past for conceiving the MAC of wireless networks. The aim of the paper is thus twofold. First, we will identify the key areas to be investigated in MAC design, and second, we will try to understand which areas can benefit from existing solutions and which oppositely require innovation in order to take into account UWB specificity. In order to reach these goals, the paper will review, for each key area, several examples of the solutions adopted in existing wireless networks. Such review, although not exhaustive, will provide the background required to analyze UWB specific topics in MAC design. It will be then shown that different areas are affected in different degrees by the adoption of UWB, ranging from cases in which existing solutions can be adopted without modifications to cases in which UWB is the key enabling technology for new MAC capabilities and innovative design, typically based on ranging and positioning.

The paper is organized as follows.

Section II describes the MAC functions required to reach the above MAC objectives, and presents extensive examples of how these functions have been accomplished in existing wireless networks. Section III focuses on the issues related to the adoption of an UWB physical layer. Conclusions are presented in Section IV. 


\section{MAC DESIGN GUIDELINES}

In this section, we will identify the key MAC design areas, corresponding to a set of MAC functions to be implemented in order to reach the key objectives discussed in Section I.

In the following, the general case of a distributed network architecture will be considered. The focus being on MAC organization, however, both centralized and distributed MAC solutions will be considered. Each of the MAC functions described below, in fact, can be implemented either in a centralized or in a distributed fashion independently on the overlaying network architecture.

MAC functions which in general need to be implemented in most systems are as follows:

a ) Medium sharing - This function determines how terminals access the medium in order to transmit packets.

b ) MAC Organization - This function deals with the organization of the network at the MAC level, i.e. how terminals coordinate themselves in resource sharing.

c ) Admission control - In Quality of Service (QoS) aware networks, this function is used to regulate the access of traffic sources to the network, avoiding congestion.

d ) Packet scheduling - When multiple traffic flows are present in the same terminal, packet scheduling is used to select the next packet to be transmitted.

e ) Power control - Power control aims at optimizing power utilization in the network.

Overlaid over the above functions is QoS management. QoS involves most of the functions defined above and can be seen as a horizontal function. For this reason, we will first address the general topic of QoS in distributed wireless networks. Then, a description of the basic MAC functions listed above will be given, and several examples of their implementation in existing wireless networks will be presented. Improvements and difficulties in the realization of such functions due to specific UWB characteristics will be highlighted.

\section{A. QoS management at the MAC Layer}

Technological progress and social modifications make data networks more and more appealing as a universal way to transfer all kinds of information. Thus a modern data network must be capable to deliver at the same time data, voice, multi-medial (e.g. streaming video), and real-time-critical traffic by adapting its behavior to different user requirements and traffic characteristics.

Voice and multi-media traffic, in particular, are characterized by requirements which are not present in non-real-time data traffic, e.g. the necessity of transferring bit streams at a minimum bit rate (determined by the application generating the traffic) with an upper bound on the end-to-end delay. The fulfillment of the above requirements guarantees that the end user perceives the offered service with the requested quality: QoS defines thus the performance which must be guaranteed by the network in order to meet user expectations.

As a consequence, the adoption of strategies, which modify network behavior depending upon traffic characteristics and QoS constraints, is commonly considered as a natural evolution of data networks.
The first step in the design of such strategies is the definition of a set of parameters defining QoS. Note that although each different service is characterized by its own application-level QoS parameters (e.g. resolution, frame rate for video services, sample rate and sample size for audio services), these are mapped onto a unique set of network-level QoS parameters, which can be listed as follows: bandwidth, end-to-end delay, jitter, bit error rate and packet loss.

Typical values of these parameters depend upon the corresponding service. A few examples are reported in Table 1 [4].

Table 1. Mapping of Services on Qos Parameters

\begin{tabular}{|c|c|c|}
\hline Service & QoS Parameter & Range \\
\hline \multirow{3}{*}{ Audio (Telephone Speech) } & Bandwidth & $16 \mathrm{kbps}$ \\
& End-to-end delay & $400 \mathrm{~ms}$ \\
& Packet loss & $10^{-2}$ \\
\hline \multirow{3}{*}{ Video (HDTV, lossy } & Bandwidth & $20 \mathrm{Mbps}$ \\
compression) & End-to-end delay & $250 \mathrm{~ms}$ \\
& Bit error rate & $10^{-6}$ \\
& Packet loss & $10^{-11}$ \\
\hline \multirow{2}{*}{ Data } & Bandwidth & $0.2-10 \mathrm{Mbps}$ \\
& End-to-end delay & $1 \mathrm{~s}$ \\
& Packet loss & $10^{-11}$ \\
\hline
\end{tabular}

Different solutions have been proposed to introduce QoS in data networks; All of these solutions rely however on the definition of a set of service classes, identifying the different levels of QoS which can be guaranteed by the network [5]. Such solutions for QoS support are defined as a component of the Network layer. Nevertheless, the effective deployment of QoS is heavily affected by the underlying Data Link and Physical layers. In the case of wireless networks, the radio transmission medium has a heavy impact on the QoS offered by the network. Network layer QoS mechanisms are in fact based on the reliability of the physical medium, something which cannot be easily guaranteed in the case of mobile terminals. Thus, the QoS concept needs to be adapted to this hostile environment.

As a matter of fact, it is impossible to guarantee at any time the fulfillment of QoS requirements at the physical layer. Any transmission medium is in fact characterized by an outage probability, defined as the out-of-service probability of the physical medium, which is different from zero. The key difference between wired and wireless networks is in the value assumed by such outage probability; In wired networks the probability of a link down is low enough to allow the upper layers to simply overlook this event. In the case of radio networks, instead, link failures are frequent enough to impact the link and network layers, and in particular the MAC sublayer, leading to the necessity of introducing mechanisms to rapidly recover errors on the link, e.g. Forward Error Correcting (FEC) codes or Automatic Repeat on reQuest (ARQ) protocols. In spite of these mechanisms, however, the fulfillment of QoS requirements cannot be guaranteed deterministically, but only with a given probability. In this perspective, the outage probability of the physical medium translates at the MAC layer in an out-of-service probability. This probability of failure, i.e. missing the QoS requirements, can be reduced by correctly designing and tuning the FEC and ARQ 
mechanisms cited above. As the channel behavior worsens, however, the fulfillment of the requirements becomes less and less realistic. The MAC may simply have not enough resource to compensate the channel outage probability.

In such a condition of scarce resource, priorities are required to obtain a fair resource sharing, at the MAC layer. Priorities can be defined at two levels:

1. priority between different users/terminals

2. priority between different traffic types (real-time/voice traffic, data traffic)

As a consequence, the introduction of QoS management involves several MAC functions, from admission control and packet scheduling, to power control and MAC organization. Examples of the impact of QoS management on the above functions will be given throughout this section.

\section{B. Medium Sharing}

Most of the existing MAC protocols for distributed networks are based on the key hypothesis that users share a single channel. From a resource sharing point of view, this implies that the resource to be shared is the radio access itself.

Two possible choices are available for resource management: terminals may contend in order to gain channel control (random access), or channel control may be granted by a control unit based on a specific resource assignment protocol (scheduled access). While the random access approach is appropriate for bursty traffic, scheduling allows a more efficient utilization of the channel when continuous streams of data packets must be transferred. Even in the case of a scheduled approach, however, a random access phase is requested, since the scheduling sequence is typically unavailable at network startup. In this section we will thus focus on available solutions for random access, while examples of scheduled access will be moved to section IIC.

Random access typical solutions for wireless networks are Aloha, Carrier Sensing Multiple Access (CSMA), and Out-ofBand signaling [2].

Aloha main advantage is simplicity. The Aloha protocol only foresees in fact a CRC field to be added to data packets before transmission. If a collision occurs, a backoff procedure is activated in order to schedule retransmission of the corrupted packet. Aloha has been proven to well behave when low traffic load is offered to the network, while performance decreases abruptly as traffic load increases and packet length grows [1]. Slotted Aloha, in which a slotted time axis is adopted, and terminals start transmission attempts only at the beginning of a time slot, improves performance without really solving this issue. For this reason, Aloha was proposed for the specific case of short, rare packet transmission (e.g. control packets), i.e. when the transmission time is low enough to mitigate the effect of collisions. Under the condition of a high traffic load, a higher throughput can be obtained by means of CSMA which is based on a channel sensing period performed by each terminal before starting transmission. The performance obtained by CSMA is however heavily affected by two phenomena, the well known "hidden terminal" and "exposed terminal" problems. In order to solve the hidden and exposed terminal problems, alternative solutions to CSMA have been proposed. The Multiple Access with
Collision Avoidance (MACA) protocol [6] for example replaces the carrier sensing procedure with a three-way hand-shake between transmitter and receiver. Following this approach, further modifications of the MACA protocol have been developed, such as MACAW [7] and MACA-By Invitation (MACA-BI) [8].

Practical implementations of MAC protocols combine handshake and carrier sensing, as proposed in the Floor Acquisition Multiple Access (FAMA) protocol [9]. These protocols are commonly referred to as CSMA with Collision Avoidance (CSMA-CA). An example of CSMA-CA is the Distributed Foundation Wireless MAC (DFWMAC) which has been adopted for the MAC layer of the 802.11 IEEE standard [10]. 802.11 adopts a Clear Channel Assessment (CCA) function which performs channel sensing in two different ways, either by measuring the received power and comparing it with a threshold, or by performing a true carrier sensing by detecting another 802.11 signal on the same channel.

An alternative solution to CSMA-CA is offered by the Out-ofBand signaling protocol [11]. This solution splits the bandwidth available for communication into two channels: a data channel used for data packet exchange, and a narrowband signaling channel on which sinusoidal signals (referred to as busy tones) are asserted by terminals which are transmitting and/or receiving in order to avoid interference produced by hidden terminals. In a distributed network, this would require each terminal which detects a transmission to transmit a busy tone to block all nodes in an area of radius $2 * \mathrm{R}$ around the transmitting node, $\mathrm{R}$ being the radio range, with the consequence of amplifying the exposed terminal problem (Fig. 1) [2], [11].

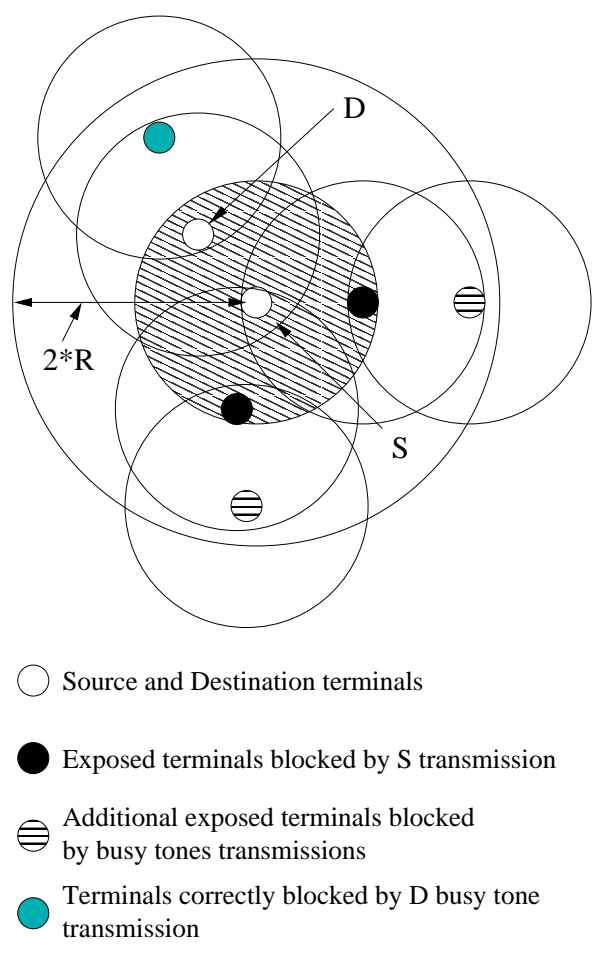

Fig. 1. Amplification of the exposed terminal problem with Out-of-Band signaling.

In order to reduce the number of exposed terminals, the use of two different Busy Tones for transmitting and receiving ter- 
minals was proposed [12].

\section{MAC Organization}

In a distributed scenario, independent terminals cooperate in order to build the network. Two main approaches are possible for network self-organization at the MAC layer: Domaindependent (clustered) and Domain-independent (flat).

Most of the MAC protocols proposed in the literature and adopted in WLAN standards rely on the explicit definition of a MAC Domain leading to a clustered network architecture, where each cluster corresponds to a MAC domain. A clustered architecture simplifies resource management within each cluster by allowing a centralized approach. Two examples of Domaindependent MAC protocols are Bluetooth and IEEE 802.15.3.

In Bluetooth [13] a Frequency Hopping - Code Division Multiple Access (FH-CDMA) scheme is adopted, and each MAC Domain is associated with a FH sequence. Terminals in a given area self-organize into MAC Domains (piconets) composed by up to eight terminals, and a centralized resource management is performed within each piconet. The set of independent piconets in the area is called scatternet.

The MAC Domain setup is started by a terminal following a neighbor discovery phase, achieved by means of a dedicated scan procedure. The terminal assumes the role of piconet Master; It determines the FH sequence, and pages one of the discovered neighbors (Slave) to start the piconet. Other devices can be included in the piconet, with individual paging by the Master. Alternatively, a device can join an active piconet by paging the Master. Since in Bluetooth the paging device always assumes the Master role, if the new paging device wants to assume a Slave role in the piconet, a Master-Slave switch procedure is required. The Master-Slave switch procedure is executed on request. No explicit criterion about when the switch should be performed is defined.

The IEEE 802.15.3 standard [14] is a second example of Domain-dependent MAC. The standard was originally developed for traditional, narrowband physical layers in the ISM band, but an UWB physical layer is currently in the standardization process. In 802.15.3, as much as in Bluetooth, the medium access is controlled in a centralized fashion within each MAC piconet. A piconet is controlled by a PicoNet Controller (PNC) which emits a periodic beacon. The channel associated with the piconet is selected based on a scanning procedure, which determines the channel subject to lower interference. No specific device is targeted in the piconet setup. It is up to the neighboring devices to join the new piconet by synchronizing to the beacon and sending an association request to the PNC by means of random packets either in a CSMA or slotted Aloha fashion. Associated devices ask for Channel Time Allocation (CTA), i.e. time slots, also by means of a CSMA protocol.

Different from the management of the Master role in Bluetooth, the PNC role in 802.15.3 is always assigned to the device with highest PNC capabilities. Each device is requested to provide its capabilities to play the PNC role during the association procedure. If a newly associated device is better suited than the current PNC, a PNC handover is performed.

The PNC and Master carry out similar tasks in the two standards as regards piconet management, including traffic schedul-
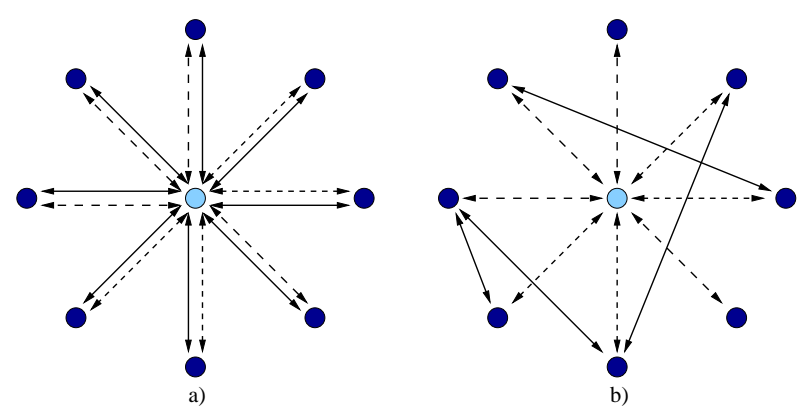

Fig. 2. Logical piconet topologies: control traffic (dashed arrows) and data traffic (filled arrows).

ing and piconet synchronization. A key difference between the two systems however is the way the data traffic flows through the piconet. In Bluetooth, direct communication between two different devices is not allowed, and the Master is in charge of relaying all traffic through the network. The piconet topology is in this case a typical star topology, as depicted in Fig. 2 a). In 802.15.3, on the contrary, the PNC only schedules CTAs to the devices, without being involved in the data packets exchange. Thus, while the piconet management is fully centralized, data transfer is performed on a peer-to-peer basis, i.e. in a pure adhoc manner (Fig. 2 b)).

The two systems, however, share the same problem of how to allow inter-piconet communication. This becomes a major issue in scalability, especially regarding traffic scheduling and routing in networks composed of a large number of piconets.

The adoption of a Domain-independent architecture avoids the problem of interpiconet communications. In a single channel scenario, a Domain-independent organization can be achieved by adopting any of the random access MAC protocols presented in section II-B. UWB radio can however provide multiple communication channels. As a consequence, we now focus on protocols designed for multiple channel networks, which have been shown to achieve better throughput in comparison with single channel solutions based on Aloha or CSMA [15]. Multiple channel solutions have been typically developed for DS-CDMA. Key concepts are however portable to UWB case.

In multiple code networks, simultaneous transmissions are allowed by using different codes for different transmissions. As a consequence, a code assignment strategy is required. Such strategy is based on one of the two following approaches:

1. Receiver-based: each receiver $j$ is characterized by a code $C_{\mathrm{j}}$, and a terminal $i$ willing to transmit to $j$ uses $C_{\mathrm{j}}$;

2. Transmitter-based: each transmitter $i$ is characterized by a code $C_{\mathrm{i}}$ and uses this code in all data transmissions.

The receiver-based strategy is far more simple from a receiver viewpoint since a receiver is required to synchronize to only one code. On the other hand, multiple transmitters directed to the same receiver, which use thus the same code for transmission, may collide. To this respect, the transmitter-based strategy is more robust, since two transmissions directed to the same receiver use different codes. In such a scenario, one of the two transmissions is perceived by the receiver as useful signal, while the other contributes to Multi-User Interference (MUI) noise. Note however that in this case the receiver must tune its hard- 
ware to the right code. Therefore this approach requires a specific code exchange procedure.

The assignment of a code to each terminal can be either static, or based on a code assignment [16]. The solution proposed in [16] guarantees that the same code is never assigned to terminals which are less than 3-hops away one from each other, avoiding thus the occurrence of collisions.

A solution based on a transmitter-oriented strategy is described in [17]. Two alternatives are proposed in order to inform the receiver of the forthcoming transmission: a common code or a receiver code. An enhancement of [17] based on the combination of handshaking and multiple codes has been recently proposed [18] As a final remark, the definition of a multiple channel MAC protocol is strictly related to the hardware complexity of the terminals. The adoption of a transmitter-based approach becomes for example straightforward if a receiver is complex enough to be capable of listening to several codes simultaneously.

The definition of MAC organization is crucial in the design of a specific MAC for UWB networks. The adoption of a Domainbased structure is a potential solution, since it addresses management of multiple Time Hopping codes. On the other hand, a multiple channel MAC could significantly increase network throughput by exploiting the inherent multiple channel UWB capability. This aspect is discussed further in Section III.

\section{Admission control}

Admission control is required when congestion must be avoided in order to meet network performance requirements. Admission control is mandatory in QoS-aware networks in which unregulated access might easily provoke violation of performance guarantees. Best effort networks do not require admission control, but can benefit from its introduction. Admission control is typically implemented with centralized schemes, as in cellular networks [19] and centralized wireless networks [20]. A few proposals for distributed schemes are however also available in the literature. These schemes rely on the cooperation of terminals in evaluating the impact of additional traffic flows on network throughput, and eventually rejecting requests causing unacceptable performance degradation typically due to MUI generated by potential new entries [21], [22], [23].

This approach is suitable for UWB networks, which rely on TH-CDMA for multiple access. A distributed admission control scheme for an UWB network is proposed in [24]. based on Bambos [21].

\section{E. Packet Scheduling}

The packet scheduling algorithm determines the order in which buffered packets are selected for transmission. In wired networks, this function has two main objectives: 1) to guarantee a fair access to the all flows to the available capacity, and 2) to support QoS if different traffic classes are present. The simplest solution is the First Come First Serve (FCFS) algorithm in which packets are sent in the same order in which they are buffered. This solution, however, provides no protection against ill-behaving sources, which can capture any percentage of the available bandwidth by increasing their packet emission rate. In order to increase fairness, a Round Robin scheme adopted to serve each traffic flow is proposed in [25]. Fair access however is not guaranteed since packets of different lengths can be present in each queue. The Weighted Fair Queueing algorithm [26] addresses this issue by assigning a weight to each queue with the aim of emulating a bit-per-bit Round Robin between different flows. In this case the introduction of QoS in the scheduling strategy is straightforward since the weights can be easily adjusted in order to take into account the QoS classes.

Efficient packet scheduling in wireless networks cannot ignore the status of the wireless channel. Several wireless scheduling algorithms, which are sensitive to channel status, have been proposed. These are based on either a simple on-off Markov channel model [27] or on more sophisticated channel models leading to accurate evaluation of the Signal-to-Noise-Ratio (SNR) [28] and external interference [29].

To this respect UWB does not present any relevant difference from other radio transmission techniques and the above protocols are directly applicable to the specific UWB case.

\section{F. Power Control}

Due to the broadcast nature of the wireless medium, the achievable performance in wireless networks strictly depends on the capability of minimizing the undesidered effects of each radio transmission on neighboring receivers. Power control leads thus to optimization of emitted power levels and achieves three desirable effects [21]: 1) Minimization of power consumption, leading to longer autonomy, 2) Reduction of interference, and 3) Adaptation of emitted power to link variations due to channel modifications and mobility.

Power control received significant attention in the last few years, in conjunction with the introduction of $3 \mathrm{G}$ cellular networks based on CDMA, since it mitigates the near-far phenomenon, in which a transmitter close to the receiver shadows the signal of a further transmitter. The centralized structure of cellular networks, however, simplifies the solution to this problem since the presence of a base-station significantly helps in the development of efficient power control algorithms. The issue is far more complicated in a distributed network architecture in which several independent links may be set up at the same time without any central controller. Nevertheless, power control should be a key property of distributed MAC protocols since it allows a significant increase in network capacity [30]. A distributed power control protocol for CDMA ad-hoc networks jointly with a power-related admission control function is proposed in [21].

Power control is important in the case of UWB networks as well, at least for two reasons: 1) UWB networks are affected by the near-far effect, although it can be expected that the high processing gain provided by the TH-IR can partially mitigates this phenomenon, and 2) the low power levels allowed for UWB communication networks impose efficiency in the use of power.

\section{THE UWB CASE}

The key functions required in the MAC design of a distributed network were described in section II. For a few of these, the use of UWB radio was investigated, either by identifying a solution proposed for the UWB case (admission control) or by explaining 
how UWB can benefit from existing solutions (packet scheduling). A more detailed analysis is required for other functions, which can be heavily affected by the adoption of UWB, such as Medium Sharing and MAC Organization. Such analysis is the main topic of section III-A. Section III-B deals with new potential MAC functions enabled by the UWB technique.

\section{A. Medium Sharing and MAC Organization in UWB networks}

The review of section II shows that the selection of channel access protocols and definition of MAC Organization are tightly related. The first step in analyzing potential MAC organization for UWB networks is thus to evaluate the applicability of existing channel access protocols to this specific case.

Several UWB definitions have been proposed in the last few years, ranging from Time Hopping Impulse Radio (TH-IR) to Direct Sequence UWB (DS-UWB), and to Multi-band UWB. In the following, we will consider TH-IR, which is the most common definition of UWB radio. In this case, the most intuitive solution is to identify each channel by a different Time Hopping code.

Independently of a selected MAC organization, that is clustered or flat, the following questions must be addressed:

- How is the channel defined for a TH-IR system?

- How is the channel accessed by terminals?

- How do terminals manage multiple channels?

It is worth noting, first, that if a Domain-based organization is adopted, the definition of intra-Domain procedures is almost independent on the channel definition. If for example a TDMA scheduled access scheme is considered, as in IEEE 802.15.3, procedures for CTA request and allocation are the same independently on the definition of the channel. Oppositely, procedures for inter-Domain communications, or channel selection, are highly dependent on channel definition.

The 802.15.3 MAC standard defines a procedure for channel selection aiming at minimizing inter-piconet interference since many interfering devices are expected to be present in the 2.4 GHz ISM band. The adoption of a UWB physical layer should amplify this issue since the UWB signal spreads over a much larger bandwidth than ISM and partially overlaps with a large number of narrowband systems. As a consequence, an accurate channel monitoring will be required in order to meet the severe coexistence issues imposed to UWB and to allow such a system to reach the requested performance. As an example of how the adoption of TH-IR would impact such procedure, if the channel is defined by means of a TH-code, coexistence with a narrowband system could be achieved by choosing a code which introduces a notch in the UWB signal Power Spectral Density in the band occupied by the narrowband system.

The channel, as defined above, must be accessed by terminals in order to exchange data and control information. The selection of the protocol to access the channel should then consider the TH-IR characteristics. The Aloha protocol requires no specific actions to be performed by the transmitter before emitting a packet. Its application to UWB is thus straightforward. As explained in section II-B the main concern about this protocol is its poor performance in heavy traffic load conditions. It should be noted, however, that the evaluation of such performance is performed under the hypothesis of destructive collisions, which is quite realistic in the case of narrowband signals, characterized by a high duty cycle. TH-IR signals, on the contrary, can achieve low duty cycles, and could thus offer a higher protection in case of packet collisions. Further research is necessary in order to better characterize UWB interference and correctly evaluate the effect of packet collisions.

The CSMA/CA protocol requires the capability of sensing the channel in order to understand if a transmission can be started. As a general remark, CSMA protocol is only suited for spread spectrum signals with low processing gain. In fact, spread spectrum systems with high processing gain do not experience significant performance increase by switching from Aloha to CSMA based on power measurement because of the lack of correlation between the interfering power measured at the transmitter and the interfering power suffered at the receiver [31]. Furthermore, true carrier sensing (i.e. the identification of another transmission) is complicated by the spreading itself, which makes it difficult to detect a spread signal, if the synchronization preamble is missed [32]. The extremely high processing gain guaranteed by TH-IR is expected to amplify these drawbacks, leading to the conclusion that CSMA is most likely not suitable for UWB systems. Let us consider for example a scenario in which two devices, $\mathrm{A}$ and $\mathrm{B}$, need to transmit data to the same receiver $\mathrm{C}$. $\mathrm{B}$ is already transmitting, while $\mathrm{A}$ is performing a carrier sensing procedure. Due to the spatial positions of $\mathrm{A}$ and $\mathrm{B}$, A does not receive any of the pulses transmitted by $\mathrm{B}$, and considers the channel as clear (Fig. 3).

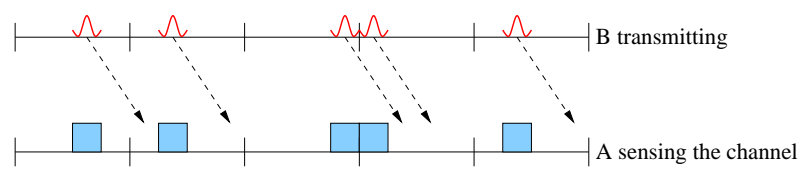

Fig. 3. Example of error in Carrier Sensing procedure in a TH-IR system.

In the worst case, A will start transmitting with the same phase as B (i.e. the same code value) and with a delay equal to the difference between the propagation delays from $\mathrm{A}$ and $\mathrm{B}$ respectively to $\mathrm{C}$ : this will lead to systematic collisions at $\mathrm{C}$ when A starts transmitting (Fig. 4).

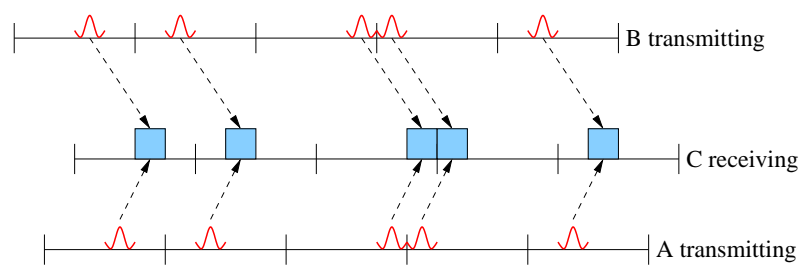

Fig. 4. Collision at the receiver due to error in Carrier Sensing procedure in a TH-IR system.

In general, the number of collisions will depend on both the relative delays between the two transmissions and the autocorrelation properties of the Time Hopping code. Simulations and measurements are required to evaluate if a simpler protocol (e.g. Aloha) can guarantee the requested performance by relying on the temporal diversity properties of TH-IR UWB and thus skipping the carrier sense procedure. 
The third random access protocol analyzed in section II-B is the Out-of-Band signaling. This solution has however several drawbacks which make its adoption in UWB networks unlikely. First, it requires additional hardware to generate one or more narrowband tones, leading thus to higher cost. Second, and most important, the propagation characteristics for narrowband tones and UWB emissions are likely to be completely different, leading to unpredictable protocol behavior due to different ranges for control and data information.

The above considerations indicate Aloha to be the best solution to allow channel access in UWB networks. This technique could be adopted in a Domain-based architecture, in which random access is only used to transfer control information and scheduled access is adopted for data transmissions. It is worth noting however that existing standards, such as 802.15.3 and Bluetooth, do not define procedures for the interconnection of independent MAC Domains. As a consequence, the maximum size of a piconet is a first bound for the maximum network size and network scalability. The same issue of course raises in the case of UWB networks, if a MAC Domain is defined. TH-IR, however, provides a built-in multiple access scheme based on TH-codes, so that the adoption of a MAC Domain is not mandatory in the design of the MAC for an UWB network. In fact, a completely distributed MAC organization can be foreseen, in which each link is activated on a different TH-code. Protocols described in section II-B for code assignment and multiple channel MAC management in CDMA networks can be adapted to the UWB, with a few additional considerations.

First, UWB systems differ from DS-CDMA, since a low duty cycle is achieved thanks to the impulsive nature of the UWB signal. This means that even if the same TH code is selected for two simultaneous transmissions, most probably, this will not lead to excessive interference because temporal separation should avoid systematic collisions. As a consequence, in network scenarios characterized by low or medium terminal densities, a solution based on a single TH code may lead to good throughput. In this case, the overhead due to a code assignment protocol may be avoided.

Second, TH-IR systems may require higher time lags for synchronization than existing DS-CDMA systems. Even if it will not hinder point-to-point communications, it should be taken into account when defining MAC and higher layer protocols. Most protocols rely in fact on the availability of a broadcast channel heard by all terminals; Each terminal must be able to synchronize to this channel within an acceptable time. This condition may not be met in TH-IR systems. In this case, alternative solutions should be adopted, either based on the absence of a broadcast channel, or based on a network reference clock maintained by all terminals in order to reduce the synchronization time to the common channel.

\section{B. UWB novel functions}

In the above sections, we analyzed how UWB may influence the implementation of traditional MAC functions. UWB unique characteristics should however enable the definition of new functions as well that are specifically designed to exploit this technology.

The main innovation offered by UWB is the capability of achieving high precision ranging. It should be noted that this characteristic is typical of spread spectrum signals in general. Time of Arrival (TOA) estimations for example can be obtained in DS-CDMA systems by evaluating time shifts between the spreading code in the receiver and the same code in the received signal. The ranging precision thus depends upon the capability of determining this time shift, and is directly related to the adopted chip rate, i.e. the spread signal bandwidth. GPS system, for example, relies on this technique, and guarantees an accuracy in TOA estimation of $100 \mathrm{~ns}$, corresponding to an accuracy of $3 \mathrm{~m}$ in distance estimation [33].

The key advantage offered by UWB is thus the ranging precision. In fact, errors in the order of centimeters can be guaranteed, much better than the precision achievable by DS-CDMA systems, thanks to a time accuracy of less than 100 picoseconds. This precision is useful in the short range scenarios (tens of meters) expected for UWB networks where positioning is effective only if high precision can be achieved.

Ranging information can be exploited in several ways in resource management. Examples are: a) Definition of distancerelated metrics for both MAC and higher layers, enabling the development of power-aware protocols, e.g. [34]; b) Evaluation of initial transmission power levels, required in distributed power control protocols [35]; c) Introduction of distributed positioning protocols in order to build a relative network map starting from ranging measurements. This map could enable location-based enhancements in several MAC and network functions, such as position-based routing, and position-aware distributed code assignment protocols in multiple channel MAC, in order to minimize MUI.

\section{CONCLUSION}

The present work reviews MAC design issues in wireless networks, with emphasis on highlighting specific design guidelines and trends for the UWB case. Key areas of MAC design were first identified, and available solutions for each area were presented through examples based on existing MAC protocols. The impact of UWB on the MAC was then investigated in a twofold way.

First, available solutions were analyzed with respect of requirements of UWB networks. The areas in which design can benefit from existing solutions and those which, oppositely, require dedicated solutions for UWB were identified. In particular, it was shown that issues related to admission control, packet scheduling and power control can be addressed by adopting similar approaches to those proposed for existing wireless networks. On the other hand, medium sharing and MAC organization require specific design in order to take into account the peculiar characteristics of UWB, such as high processing gain and high synchronization latencies.

Finally, new MAC functions enabled by specific UWB properties, in particular precise ranging, were identified. Examples of how these new functions may improve MAC performance were suggested. Future work will address the complete definition and algorithmic implementation of the above functions. 


\section{ACKNOWLEDGMENTS}

This work was partially supported by the European Union under project $n^{\circ}$ IST-2001-32710-U.C.A.N.

\section{REFERENCES}

[1] D. Bertsekas and R. Gallager, "Data Networks," 2nd edition, Prentice Hall, 1992.

[2] A. Chandra, V. Gummalla and J.O. Limb, "Wireless Medium Access Control Protocols," http://www.comsoc.org/pubs/surveys, Second quarter 2000.

[3] H. Choi and N. Moayeri, "Evaluation procedure for 802.16 MAC Protocols," http://grouper.ieee.org/groups/802/16/tg1/mac/pres/802161mp00_16.pdf.

[4] K. Nahrstedt and R. Steinmetz, "Resource Management in Networked Multimedia Systems," IEEE Computer, vol. 28, no. 5, 52-63, May 1995.

[5] X. Xiao and L.M. Ni, "Internet QoS: A Big Picture," IEEE Network, vol. 13, no. 2, 8-18, March/April 1999.

[6] P. Karn, "MACA-A new Channel Access Protocol for Packet Radio," Proceedings of the ARRL/CRRL Amateur Radio Ninth Computer Networking Conference, 134-140, 1990.

[7] V. Bharghavan, A. Demers, S. Shenker and L. Zhang, "MACAW: A medium access protocol for wireless LANs," Proceedings of SIGCOMM '94, 212-225, 1994.

[8] F. Talucci and M. Gerla, "MACA-BI (MACA By Invitation): A wireless MAC protocol for high speed ad hoc networking," Proceedings of Universal Personal Communications 1997 IEEE 6th International Conference on, vol. 2 , 913-917, 12-16 October 1997.

[9] C.L. Fullmer and J.J. Garcia-Luna-Aceves, "Floor acquisition Multiple Access (FAMA) for Packet Radio Networks," Proceedings of the conference on Applications, Technologies, Architectures and Protocols for Computer Communication (SIGCOMM '95), 262-273, 1995.

[10] B.P. Crow, I. Widjaja, J.G. Kim and P.T. Sakai, "IEEE 802.11: Wireless Local Area Networks," IEEE Commn. Mag., vol. 35, no. 9, 116-26, September 1997.

[11] F.A. Tobagi and L. Kleinrock, "Packet Switching in Radio Channels: Part II - The Hidden Terminal Problem in Carrier Sense Multiple Access and the Busy Tone Solution," IEEE Trans. Commun., COM-23, 1417-33, 1975.

[12] J. Deng and Z.J. Haas, "Dual Busy Tones Multiple Access (DBTMA): A New Medium Access Control for Packet Radio Networks," Universal Personal Communications IEEE 1998 International Conference on, vol. 2, 973-977, 5-9 October 1998.

[13] J.C. Haartsen, "The Bluetooth Radio System," IEEE Personal Communications, 28-36, February 2000.

[14] "IEEE 802.15.3 MAC standard," available at http://www.ieee.org/.

[15] T. Makansi, "Transmitter-Oriented Code Assignment for Multihop Radio Networks," IEEE Trans. Computers, COM-35(12), 1379-1382, December 1987.

[16] J.J. Garcia-Luna-Aceves and J. Raju, "Distributed Assignment of codes for multihop packet-radio networks," Proceedings of IEEE MILCOM 97, vol. 1, 450-454, 2-5 November 1997.

[17] E.S. Sousa and J.A. Silvester, "Spreading Code protocols for Distributed Spread-Spectrum Packet Radio Networks," IEEE Trans. Commun., COM$36,272-281,1988$

[18] M. Joa-Ng and I. Lu, "Spread Spectrum Medium Access Protocol with Collision Avoidance in Mobile Ad-hoc Wireless Network," Proceedings of IEEE INFOCOM 1999, 776-783, April 1999.

[19] M. Mouly and M.B. Pautet, "The GSM System for Mobile Communication," CellSys, 1992.

[20] J. Khun-Jush, G. Malmgren, P. Schramm, and J. Torsner, "Overview and performance of HIPERLAN type 2-a standard for broadband wireless communications," Proceedings of IEEE 51 st Vehicular Technology Conference, vol. 1, 112-117, 15-18 May 2000.

[21] N. Bambos, "Toward power-sensitive network architectures in wireless communications: Concepts, issues, and design aspects," IEEE Pers. Commun., vol. 5, 50-59, June 1998.

[22] S. Valaee and B. Li, "Distributed Call Admission control for Ad-hoc networks," Proceedings of IEEE 56th Vehicular Technology Conference, vol. 2, 1244-1248, 24-28 September 2002.

[23] Y. Yang and R. Kravets, "Contention-Aware Admission Control for Ad Hoc Networks," University of Illinois in Urbana-Champaign Technical Report UIUCDCS-R-2003-2337, April 2003.

[24] F. Cuomo, C. Martello, A. Baiocchi and F. Capriotti, "Radio Resource Sharing for Ad Hoc Networking with UWB," IEEE Journal on Selected Areas in Communications, vol. 20, no. 9, 1722-1732, December 2002.
[25] J. Nagle, "On Packet Switches with Infinite Storage," IEEE Trans. Comm., vol. 35, no. 4, 435-438, April 1987.

[26] A. Demers, S. Keshav, and S. Shenker, "Analysis and Simulation of a Fair Queueing Algorithm," Proceedings of ACM SIGCOMM, 1-12, Austin, Texas, September 1989.

[27] S. Lu, V. Bharghavan and R. Srikant, "Fair scheduling in wireless packet networks," IEEE/ACM Transactions on Networking, vol. 7, no. 4, 473489, August 1999.

[28] H. Aida, Y. Tamura, Y. Tobe, and H. Tokuda, "Wireless packet scheduling with signal-to-noise ratio monitoring," Proceedings of IEEE Conference on Local Computer Networks, 32-41, 8-10 November 2000.

[29] N. Golmie, N. Chevrollier, and I. ElBakkouri, "Interference aware Bluetooth packet scheduling," Proceedings of IEEE Global Telecommunications Conference, vol. 5, 2857-2863, 25-29 November 2001.

[30] P. Gupta and P.R. Kumar, "The capacity of wireless networks," IEEE Transactions on Information Theory, vol. 46, no. 2 , 388-404, March 2000.

[31] E.S. Sousa, "On channel power sensing in terrestrial spread spectrum packet radio networks," Proceedings of IEEE INFOCOM 1989, vol. 3, 1072-1077, 23-27 April 1989.

[32] R.E. Kahn, "Gronemeyer S. A., Burchfiel J, and Kunzelman R.C. (1978), "Advances in Packet Radio Technology," Proceedings of the IEEE, vol. 66, no. 11, 1468-1496, November 1978

[33] I.A. Getting, "The Global Positioning System," IEEE Spectrum, 36-38, 43-47, December 1993.

[34] P. Baldi, L. De Nardis and M.-G. Di Benedetto, "Modeling and Optimization of UWB Communication Networks through a flexible cost function," IEEE Journal on Selected Areas in Communications, vol. 20, no. 9, 17331744, December 2002

[35] A.J. Goldsmith and S.B. Wicker, "Design Challenges for EnergyConstrained Ad-Hoc Wireless Networks," IEEE Wireless Communications, vol. 9, no. 4, 8-27, August 2002.

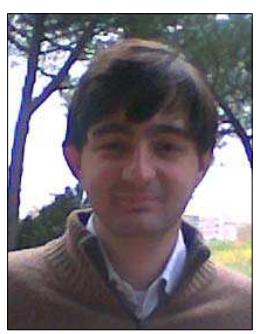

Luca De Nardis received his "Laurea" degree in telecommunications engineering from the University of Rome La Sapienza in 2001 with a thesis on wireless network topologies in the framework of the European project whyless.com. Since 2002 he has been participating in the European project UCAN. Currently, he is a Ph.D. student in Information and Communication Theory in the INFOCOM Department of the University of Rome. His research focuses on UWB technology, ad-hoc communication networks organization, Medium Access Control and routing protocols

for wireless networks.

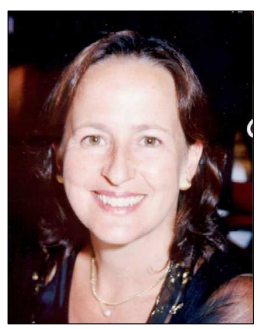

Maria-Gabriella Di Benedetto obtained her Ph.D. in Telecommunications in 1987 from the University of Rome La Sapienza, Italy. In 1991, she joined the Faculty of Engineering of University of Rome La Sapienza, where currently she is a Full Professor of Telecommunications at the Infocom Department. She has held visiting positions at the Massachusetts Institute of Technology, the University of California, Berkeley, and the University of Paris XI, France. In 1994, she received the Mac Kay Professorship award from the University of California, Berkeley. Her research interests include speech analysis and synthesis, and digital communication systems. From 1995 to 2000, she directed four European projects for the design of UMTS. Since 2000 she has been active in fostering the development of Ultra Wide Band (UWB) radio communications in Europe. She is currently the director for the Infocom Dept. of two European projects (whyless.com and UCAN) aimed at the design and implementation of UWB ad-hoc networks. Within the forthcoming 6th EU Framework her "Networking with UWB" research group will participate to the Pulsers Project which will integrate UWB research and development in Europe for the next years. Dr. Di Benedetto was co-editor for IEEE JSAC of a Special Issue on UWB Radio in Multi-Access Wireless Communications (December 2002). 